
M. Lommel, $\mathrm{PhD}$
S. Cirak, MD
T. Willer, PhD
R. Hermann, MSc
G. Uyanik, MD
H. van Bokhoven, PhD
C. Körner, PhD
T. Voit, MD
I. Barić, $\mathrm{MD}$
U. Hehr, MD
S. Strahl, $\mathrm{PhD}$

Address correspondence and reprint requests to Prof. Dr. Sabine Strahl, Heidelberg Institute for Plant Sciences, Dept. V Cell Chemistry, University of Heidelberg, Im Neuenheimer Feld 360, D-69120 Heidelberg, Germany

sstrahl@hip.uni-heidelberg.de

Supplemental data at www.neurology.org

\section{Correlation of enzyme activity and clinical phenotype in POMT1- associated dystroglycanopathies}

\section{ABSTRACT}

Background: Mutations in protein O-mannosyltransferases (POMTs) cause a heterogeneous group of muscular dystrophies with abnormal glycosylation of $\alpha$-dystroglycan (dystroglycanopathies). The wide spectrum of clinical severities ranges from Walker-Warburg syndrome (WWS), associated with brain and eye abnormalities, to mild forms of limb girdle muscular dystrophy (LGMD).

Objective: The aim of this study was to elucidate the impact of mutations in POMT1 on the clinical phenotype.

Methods: We examined 2 patients with POMT1-associated $\alpha$-dystroglycanopathy, 1 displaying a LGMD2K and 1 with a WWS phenotype. Using dermal fibroblasts, we analyzed the influence of the POMT1 mutations on the glycosylation status of $\alpha$-dystroglycan, protein O-mannosyltransferase activity, and the stability of the mutant POMT1 protein.

Results: We report on novel compound heterozygous mutations in POMT1 (p.L171A and p.A589VfsX38) that result in LGMD2K. We further demonstrate that a homozygous splice site mutation of a recently identified WWS patient results in POMT1 p.del77-93. Using dermal fibroblasts, we show that mannosyltransferase activity is reduced in the patients and that stability of POMT1 mutant proteins p.A589VfsX38 and p.del77-93 is significantly decreased.

Conclusions: Our results suggest that dermal fibroblasts can be applied to facilitate the diagnostic analysis of dystroglycanopathy patients as well as to study the pathogenic mechanism of POMT mutations. Characterization of the POMT1 substrate protein $\alpha$-dystroglycan and POMT in vitro mannosyltransferase activity shows that the severity of the clinical phenotype of the patients analyzed is inversely correlated with POMT activity. Neurology ${ }^{\circledR}$ 2010;74:157-164

\section{GLOSSARY}

$\boldsymbol{\alpha}$-DG $=\alpha$-dystroglycan; CMD $=$ muscular dystrophies; LGMD = limb girdle muscular dystrophy; MLPA = multiplex ligationdependent probe amplification; $\mathbf{P O M T}=$ protein O-mannosyltransferase; $\mathbf{W W S}=$ Walker-Warburg syndrome.

In humans, impaired protein O-mannosylation results in congenital muscular dystrophies (CMD) that are referred to as secondary $\alpha$-dystroglycanopathies since a common pathologic feature is the hypoglycosylation of $\alpha$-dystroglycan $\left(\alpha\right.$-DG). ${ }^{1}$ The most severe disorder is Walker-Warburg syndrome (WWS, OMIM 236670), characterized by CMD associated with severe brain malformations and ocular abnormalities. ${ }^{2}$ WWS patients often die within the first year of life. In contrast, the mildest disorders may not present until adulthood, such as limbgirdle muscular dystrophy (LGMD), where neither brain nor eyes are affected. ${ }^{2}$ In between these extremes, intermediate phenotypes have been described with CMD but mild mental retardation and microcephaly.

\footnotetext{
From the Heidelberg Institute for Plant Sciences, Dept. V Cell Chemistry (M.L., T.W., S.S.), University of Heidelberg, Germany; Institute of Child Health (S.C.), Dubowitz Neuromuscular Centre, University College London, London, UK; Zentrum fuer Kinderheilkunde (R.H., T.V.), Peadiatrie 1, Universitaetsklinikum Essen, Germany; Institute for Human Genetics (G.U.), University Medical Center Hamburg-Eppendorf, Hamburg, Germany; Department of Human Genetics (H.v.B.), Radboud University Nijmegen Medical Center, Nijmegen, the Netherlands; Zentrum fuer Kinder- und Jugendmedizin (C.K.), Kinderheilkunde I, Universitaetsklinikum Heidelberg, Germany; Department of Pediatrics (I.B.), University Hospital Center and School of Medicine, Zagreb, Croatia; Center for Human Genetics (U.H.), Regensburg, Germany. T.W. is currently affiliated with Department of Molecular Physiology \& Biophysics, The University of Iowa Carver College of Medicine, IA. T.V. is currently affiliated with Institut de Myologie, Université Pierre et Marie Curie Paris 6, UPMC UMR S 974, INSERM U 974, CNRS UMR 7215, Groupe Hospitalier PitiéSalpêtrière, APHP, Paris, France.

Disclosure: Author disclosures are provided at the end of the article.
} 
The protein O-mannosyltransferases POMT1 and POMT2 initiate the biosynthesis of O-mannosyl glycans in the endoplasmic reticulum. ${ }^{3}$ Mutations in the POMT1 (OMIM 607423) and POMT2 (OMIM 607439) genes result in $\alpha$-dystroglycanopathies with overlapping phenotypes. Distinct mutations in POMT1 as well as POMT2 have been identified in patients with WWS, muscle-eye-brain disease (OMIM 253280), and mild forms of CMD such as congenital muscular dystrophy with mental retardation (CMD/MR), LGMD2K (OMIM 609308), and LGMD2N. ${ }^{4-11}$ Based on these observations, an inverse correlation between the degree of the remaining POMT activity and the severity of the clinical phenotype has been postulated. ${ }^{7}$ However, so far, direct evidence to support this hypothesis is lacking.

In this study, we determine POMT O-mannosyltransferase activity in dermal fibroblasts of patients with different mutations in the POMT1 gene. Our data show an inverse correlation between POMT activity and the severity of the clinical phenotype in the patients analyzed.

METHODS Patients. Patient 1 was originally identified as LGMD2K. The 10-year-old boy was born at term after an uneventful pregnancy. Developmental milestones were delayed with independent walking at 22 months. He developed secondary microcephaly with occipitofrontal circumference of $49 \mathrm{~cm}$ $(0.7 \mathrm{~cm}$ below third centile) at 6 years of age and is mentally retarded with an IQ of 68 (80\% interval, 63-79) according to the Snijders-Oomen scale. ${ }^{12}$ Brain MRI scans did not reveal structural changes. Serum creatine kinase levels were increased $(10 \times$ normal $)$ and hypertrophy of the calf muscle and Gowers sign with limb-girdle weakness was observed at 5 years. The muscle biopsy at 3 years of age showed a myopathic pattern and staining with a core antibody against $\alpha-\mathrm{DG}^{13}$ revealed a patchy reduction of dystroglycan expression (data not shown).

Patient 2 presented with typical clinical signs of WWS. A description can be found elsewhere..$^{14}$

Cell lines and mutation analyses. Primary dermal fibroblasts and peripheral blood samples were obtained after informed consent from the parents. Fibroblasts were grown in Dulbecco modified Eagle's medium supplemented with $10 \% \mathrm{fe}$ tal calf serum, $60 \mu \mathrm{g} / \mathrm{mL}$ penicillin, and $100 \mu \mathrm{g} / \mathrm{mL}$ streptomycin. Cell lines were cultivated at $37^{\circ} \mathrm{C}$ in a $5 \% \mathrm{CO}_{2}$ incubator.

DNA was extracted from blood using standard protocols. The 18 coding exons (exons 2-20) and intron-exon boundaries of the POMT1 gene were amplified by PCR., ${ }^{5,14}$ PCR products were sequenced using the ABI Prism BigDye Terminator v1.1 Cycle Sequencing Kit and analyzed on an ABI 3100 Avant sequencer (Applied Biosystems).

Multiplex ligation-dependent probe amplification (MLPA; MRC-Holland) analysis for POMT1 was performed according to manufacturer's instructions. The kit contains probes for POMT1 exons 2, 5, 17, and 19.

RT-PCR analyses. Total RNA extracts were reverse transcribed using the Omniscript reverse transcriptase kit (Qiagen) and oligo-dT primers. POMT1 cDNA was amplified by nested PCR. Primer sequences are listed in table e-1 on the Neurology ${ }^{\circledR}$ Web site at www.neurology.org. PCR products were separated on $1.5 \%$ agarose gels or cloned into a TOPO-TA Cloning Vector (Invitrogen) for sequencing.

Antibody production. Anti-POMT1 antiserum was produced using a synthetic peptide corresponding to aa Arg-249 to His-364 of mouse POMT1. The peptide was coupled to keyhole limped hemocyanin and injected into rabbits. Antibodies were purified using the peptide bound to cyanogen bromide-activated Sepharose. Peptide synthesis, coupling, immunizations, and affinity purifications were done at Pineda Antikoerper Service (Berlin/Germany).

Isolation of microsomal membrane fractions. Microsomal membrane fractions from cultured cells were isolated as described previously. ${ }^{15}$ Microsomal membranes were resuspended in $20 \mathrm{mM}$ Tris- $\mathrm{HCl} \mathrm{pH}$ 8.0, $10 \mathrm{mM}$ EDTA. Protein concentration was determined using the $\mathrm{D}_{\mathrm{c}}$-Protein Assay (BioRad).

Wheat germ agglutinin-glycoprotein enrichment. Cultured fibroblasts were washed with PBS containing protease inhibitors and harvested in the same buffer. Cell pellets were processed as described elsewhere. ${ }^{16}$

Western blot analyses. Microsomal membranes (180 $\mu$ g protein) or wheat germ agglutinin-enriched glycoprotein fractions were separated on 8\% SDS-polyacrylamide gels and transferred to nitrocellulose. Polyclonal anti-POMT1, monoclonal anti- $\alpha$-dystroglycan (IIH6, Upstate), and polyclonal anti- $\beta$-dystroglycan (C20, Santa Cruz Biotechnologies Inc.) antibodies were used. After decoration with peroxidase-coupled secondary antibodies immunoreactivity was visualized by chemiluminescence.

Assay for protein O-mannosyltransferase activity. Protein O-mannosyltransferase activity was determined based on the amount of $\left[{ }^{3} \mathrm{H}\right]$-mannose transferred from Dol-P- $\left[{ }^{3} \mathrm{H}\right]$-mannose to an $\alpha$-DG glutathione- $S$-transferase fusion as described elsewhere. ${ }^{15}$

RESULTS Two novel compound heterozygous POMT1 mutations result in LGMD2K phenotype. Patient 1 was originally identified as LGMD with mental retardation (for details, see Methods). Sequencing of all coding exons of POMT1 revealed a novel heterozygous missense mutation in exon 6 (data not shown) predicting a substitution of the conserved leucine residue Leu171 to alanine (p.L171A; NCBI reference sequence: NP_009102.3) in the POMT1 protein (figure 1A). This substitution was not observed in 51 healthy control subjects of European descent, and is not present in SNP databases or human EST clones. Sequencing of the coding exons of POMT2 and FKRP did not reveal any mutations. Thus, we performed MLPA-analysis for POMT1 which identified a partial heterozygous deletion at the exon 19 probe (data not shown). For further refinement, we performed RT-PCR analyses of POMT1 transcripts. Total RNA from patient 1 and a healthy control was reverse transcribed with oligo-dT 
A

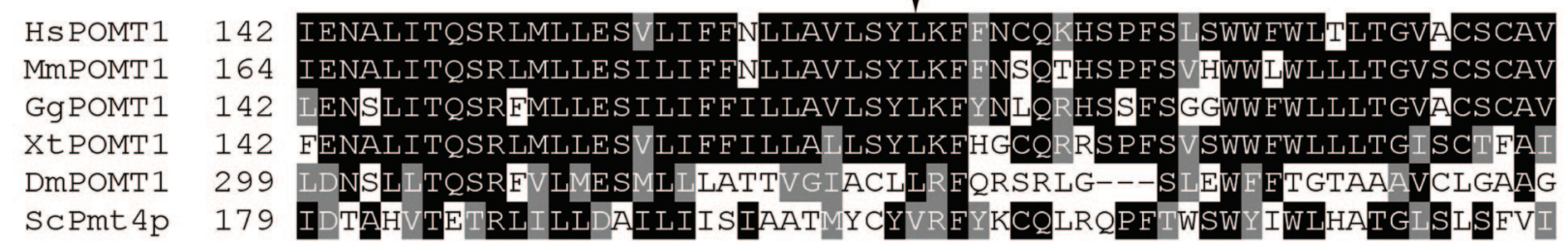

B
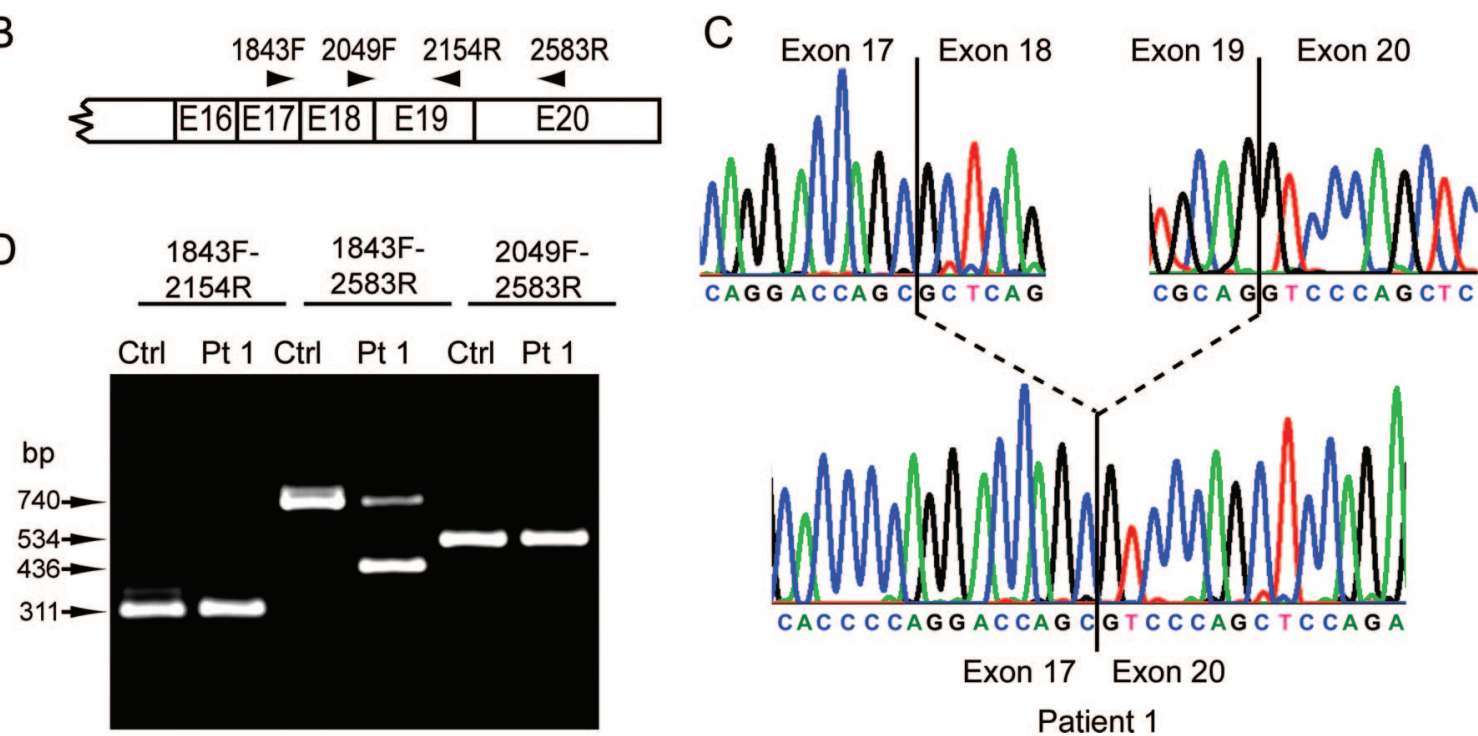

$740 \mathrm{bp}$ fragment

Patient 1

(A) Multiple sequence alignment, generated with the Clustal W algorithm ${ }^{27}$ using protein sequences of the following PMT4 subfamily ${ }^{19}$ members: Homo sapiens POMT1 (NP_009102.3), Mus musculus POMT1 (NP_660127.1), Gallus gallus POMT1 (NP_001025856.1), Xenopus tropicalis (NP_001006756), Drosophila melanogaster POMT1 (NP_524025.2), and Saccharomyces cerevisiae PMT4 (NP_012677.1). The black arrow indicates the position of the p.L171A mutation that was detected in patient 1. Identical aa are shown in black, similar aa in gray. (B) Schematic representation of the human POMT1 cDNA covering the region from exon 16 to exon 20. Positions of the primers used in (D) are indicated. (C) Sequencing of the 740-bp and 436-bp PCR fragments obtained from patient 1 confirm the absence of exon 18 to exon 19 from the smaller PCR product. (D) Amplification of the cDNA region containing exon 18 to exon 19 results in a 740 -bp and 436-bp PCR product (primers 1843F/2583R) indicating a heterozygous deletion of these exons in patient 1.

primers. PCR reactions using primer combinations that cover the POMT1 cDNA coding exons resulted in products of the expected size (figure $1, \mathrm{~B}$ and $\mathrm{D}$ ). However, amplification of the cDNA spanning exon 18 and exon 19 (primers 1843F/2583R, figure 1B) besides the expected 740 bp product resulted in an additional PCR fragment of approximately $440 \mathrm{bp}$ only in the patient (figure 1D). The observed 300-bp difference between these PCR products suggested a heterozygous deletion of exon 18 to exon 19 in the patient. Sequencing of the PCR products verified this deletion (figure 1C). The lack of exon 18 to exon 19 in the POMT1 transcript results in a frameshift mutation, causing an amino acid change at position 589 from alanine to valine, followed by a premature stop codon after 38 amino acids (p.A589VfsX38).

A homozygous splice site mutation causes deletion of exon 4 in POMT1-WWS patient. Patient 2 was recently identified as WWS carrying a homozygous base substitution at the 5 -splice donor site of intron
4 (c. $280+1 \mathrm{G}>\mathrm{T}$; NM_007171). ${ }^{14}$ This mutation is likely to impair the correct splicing of the POMT1 precursor mRNA. To further analyze the hypothesized splicing defect, we characterized the POMT1 cDNA amplified from dermal fibroblasts of patient 2 and 2 control cell lines. PCR analysis using a primer combination flanking exon 4 (tw297/tw298; figure 2A) resulted in the expected 252 bp fragments in the control cDNAs, whereas in the WWS patient the detected fragment is approximately 50 bp smaller (figure 2B, upper panel). The observed difference in the length of the PCR products suggested a deletion of exon 4 (51 bp) in the patient's mRNA. Deletion of exon 4 is further supported by the fact that a RTPCR reaction using 1 primer within exon 4 (tw296 and tw298; figure 2A) resulted in the expected 197 bp POMT1 PCR fragment in the control cDNA, but was absent in the patient (figure $2 \mathrm{~B}$, lower panel). Sequence analysis of the $201 \mathrm{bp}$ PCR fragment (tw297 and tw298; figure 2, A and B) verified the 


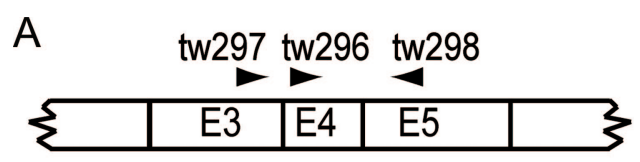

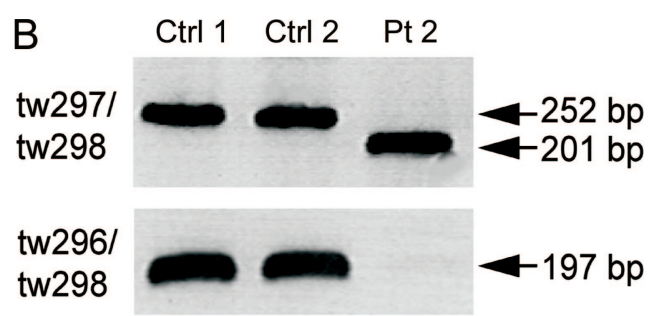

C

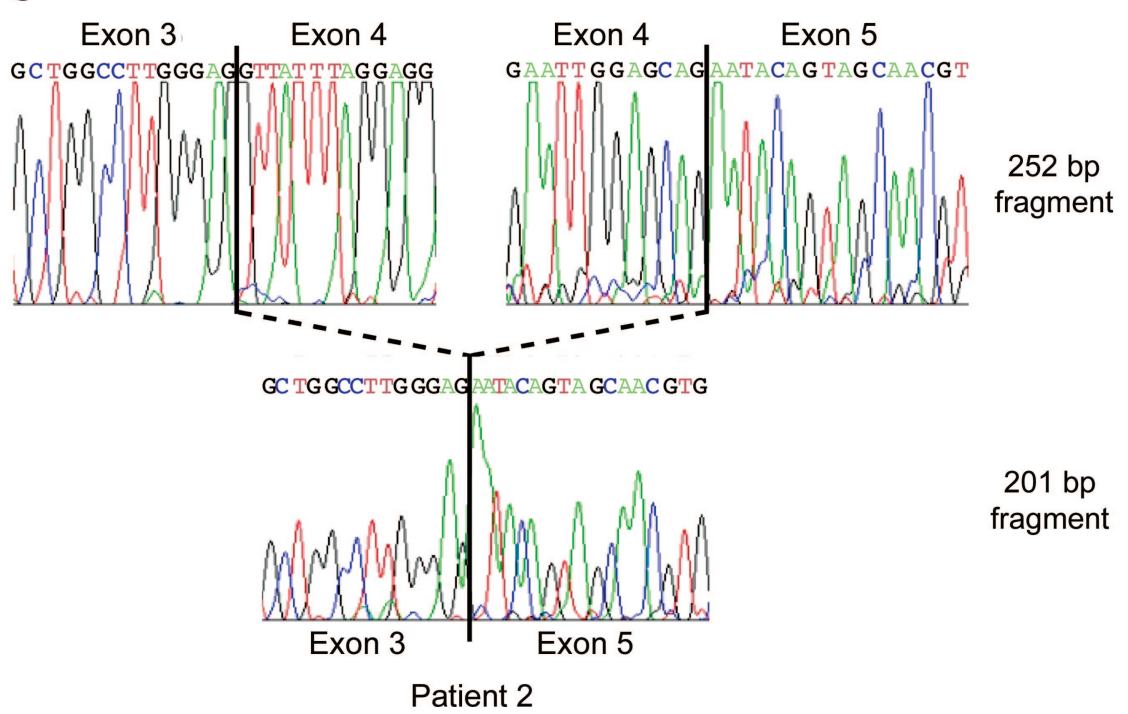

(A) Schematic representation of the applied RT-PCR strategy. (B) c. $280+1 \mathrm{G}>\mathrm{T}$ results in skipping of exon 4. PCR with primers tw297/tw298 produces a 252-bp fragment using cDNA derived from 2 control cell lines and a 201-bp in cDNA prepared from patient 2. The absence of the 197-bp RT-PCR product of patient 2 upon amplification with the primer combination tw296/tw298 confirmed the absence of exon 4. (C) Sequencing of the 201-bp PCR fragment obtained in patient 2 confirmed the absence of exon 4 from the smaller PCR product.

deletion of exon 4 (figure 2C). Taken together, the mutation of the $5^{\prime}$-splice donor site of intron 4 causes skipping of exon 4 during processing of the POMT1 precursor mRNA. Deletion of exon 4 results in the loss of 17 amino acids (p.del77-93) within the putative catalytic domain between the first and the second transmembrane domain of the POMT1 protein.

Glycosylation of $\alpha$-DG and POMT O-mannosyltransferase activity correlate with the phenotype of the secondary dystroglycanopathy. Both the LGMD2K and the WWS patient carry POMT1 mutations; however, they both resemble opposite ends of the clinical spectrum within the secondary $\alpha$-dystroglycanopathies. To characterize in more detail how the respective POMT1 mutations contribute to the clinical picture, we analyzed the $\mathrm{O}$ mannosylation status of the POMT1 substrate $\alpha$-DG. Wheat germ agglutinin glycoproteinenriched fractions prepared from dermal fibroblast cell lines were analyzed by Western blot. Antibodies directed toward a so far unidentified O-mannosyllinked glyco-epitope on $\alpha$-DG (IIH6) detected
$\alpha$-DG variants between 100 and $125 \mathrm{kDa}$ in control cells (figure 3; lane 1). Similar variants were detected in extracts derived from the LGMD2K patient but to a highly reduced amount (figure 3; lane 2). In contrast, no IIH6 positive $\alpha$-DG was detectable in the WWS patient (figure 3; lane 3). Equal loading was confirmed by detection of $\beta$-dystroglycan. These findings confirm a direct correlation between the severity of the clinical phenotype and the degree of functionally $\mathrm{O}$-mannosylated $\alpha$-DG.

We further analyzed whether differences in the $\mathrm{O}$-mannosylation state of $\alpha$-DG are reflecting differences in POMT activity in patient cells. To this end, we measured in vitro POMT mannosyltransferase activity in dermal fibroblasts of the patients. First, we adapted an enzymatic assay that was previously described for human embryonic kidney fibroblast (HEK 293) cells and lymphoblasts. ${ }^{15,17}$ POMT activity in human dermal fibroblasts highly exceeds the activity in HEK 293 cells (data not shown). ${ }^{15} \mathrm{~A}$ linear increase in the transfer of radioactive mannose to the mannosyl acceptor was observed within the range of $20 \mu \mathrm{g}$ to $120 \mu \mathrm{g}$ of microsomal membranes (fig- 


\section{Figure 3 O-mannosylation of $\alpha$-DG in POMT1 \\ associated $\alpha$-dystroglycanopathies}

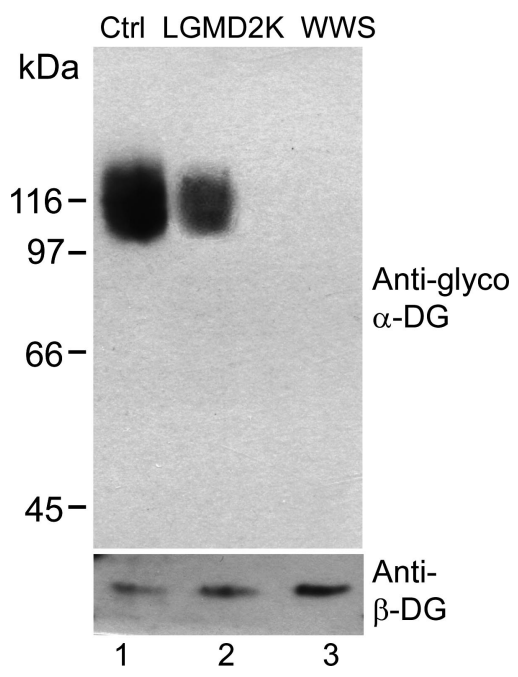

Western blot analysis of wheat germ agglutinin-glycoprotein enriched extracts prepared from control, LGMD2K, and Walker-Warburg syndrome (WWS) patient-derived dermal fibroblasts. Blots were probed with IIH6-antibodies that specifically recognize an O-mannosyl linked glycoepitope on $\alpha$-DG (anti-glyco $\alpha$-DG). To ensure equal protein loading we analyzed an identical blot was probed with $\beta$-dystroglycan specific antibodies (anti- $\beta$-DG).

ure $4 \mathrm{~A})$. To ensure that the mannose donor is not limiting to the reaction, increasing amounts of DolP-Man were added. As shown in figure 4A, excess of the mannosyl donor was achieved between 1 pmol and 2 pmol. According to these results, enzyme activity was determined using 2 pmol of Dol-P- $\left[{ }^{3} \mathrm{H}\right] \mathrm{Man}$ and $80 \mu \mathrm{g}$ of microsomal membranes derived from fibroblasts of controls and patients. Although POMT activities among the tested control cell lines showed some variability, a clear reduction was observed in the analyzed patients. When compared to the controls, $\sim 40 \%$ residual activity was detected in the LGMD2K patient (figure 4B). In contrast, in the WWS patient described above (POMT1 p.del77-93) only $6 \%$ residual POMT activity was detected. An additional WWS patient ${ }^{4}$ (POMT1 mutation: G76R; NP_009102.3) showed $\sim 10 \%$ residual POMT activity.

Our data show that the degree of $\alpha$-DG O-mannosylation and the enzymatic activity of the mutated POMT1 protein inversely correlate with the phenotypic severity observed in the patients analyzed.

Amino acid deletions affect stability of mutant POMT1 proteins. Since POMT1 mutations differently affect enzymatic activity, we further analyzed their impact on the POMT1 protein. We performed Western blot analysis of microsomal membranes from control and patient-derived dermal fibroblast cells. For POMT1 detection, we generated antibodies directed against a 15 amino acid peptide (Arg-249 to His-364) located in the central hydrophilic domain of mouse POMT1 (described in Methods). This domain is not affected in the analyzed POMT1 patients. As shown in figure 5 (lane 1), on Western blot wild-type POMT1 can be detected with an apparent molecular weight of $85 \mathrm{kDa}$. In accordance with the two POMT1 mutant alleles, 2 proteins are observed in the LGMD2K patient. The $85 \mathrm{kDa}$ protein corresponds to the POMT1 p.L171A amino acid substitution, whereas the $71 \mathrm{kDa}$ protein represents the p.A589VfsX38 mutant form of POMT1 (figure 5; lane 2). Comparing the quantities of the 2 proteins, the amount of p.A589VfsX38 POMT1 appears to be significantly lower, suggesting decreased stability of the mutant protein. Remarkably, in the WWS patient POMT1 p.del77-93 could not be detected (figure 5; lane 3), although the mutant protein lacking amino acids 77-93 does contain the antiPOMT1 epitope. Our data show that deletion of different regions of POMT1 affects protein stability. In contrast, amino acid substitution p.L171A results in a stable but less active POMT1 protein coinciding with the milder phenotype of the LGMD2K patient.

DISCUSSION We identified 2 novel POMT1 mutations that in combination result in LGMD2K phenotype (figure 1). Mutation p.A589VfsX38 causes deletion of the C-terminal 158 amino acids including 2 putative transmembrane spans and the loop6 domain (figure e-1). This deletion significantly affected POMT1 protein stability (figure 5). In contrast, amino acid substitution p.L171A resulted in decreased O-mannosyltransferase activity without affecting POMT1 stability (figures 4 and 5). Leu171 is conserved in vertebrate and invertebrate POMT1s (figure 1A) which provides further pathologic evidence. In addition, we characterized a POMT1 mutation p.del77-93 that results in WWS ${ }^{14}$ (figure 2). This mutation has also been reported in 2 other WWS patients. ${ }^{18}$ The POMT1 p.del77-93 protein lacks 17 amino acids within a hydrophilic domain between 2 putative transmembrane spans close to the $\mathrm{N}$-terminus of the protein (figure e-1). This region has been demonstrated to be essential for protein O-mannosyltransferase activity in yeast. ${ }^{19}$ Western blot analyses suggest that the mutation affects protein stability and that the POMT1 p.del77-93 mutant protein is degraded in the patient (figure 5).

We determined POMT activity in dermal fibroblast cells of these patients. In agreement with the lack of the POMT1 protein, the WWS patient (figures 3 and 4) is characterized by abolished in vitro as 

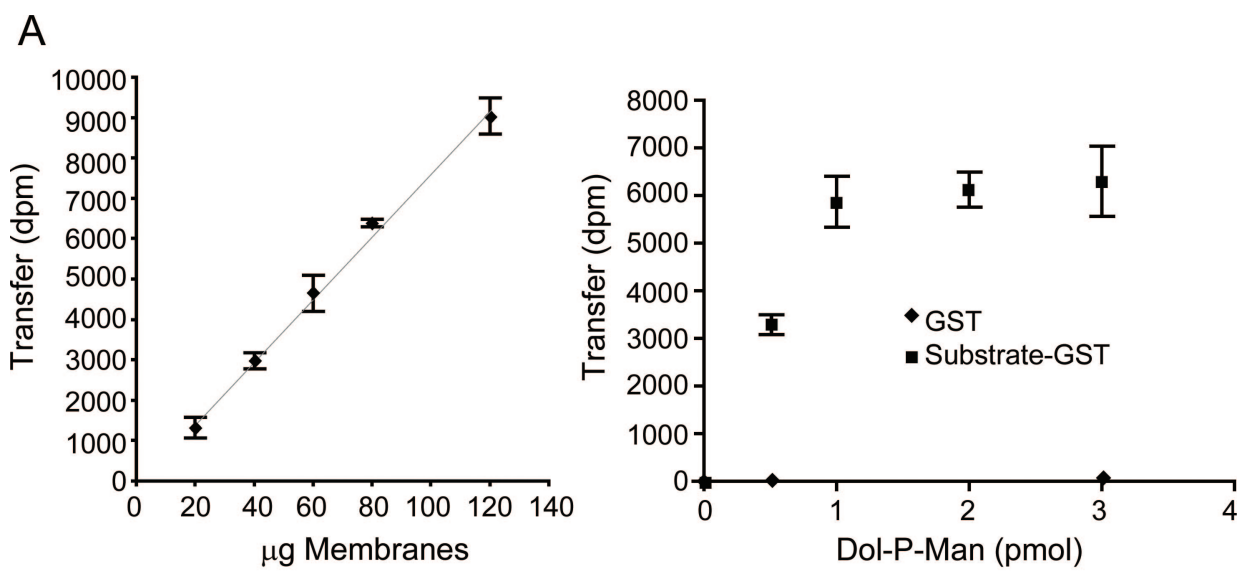

B

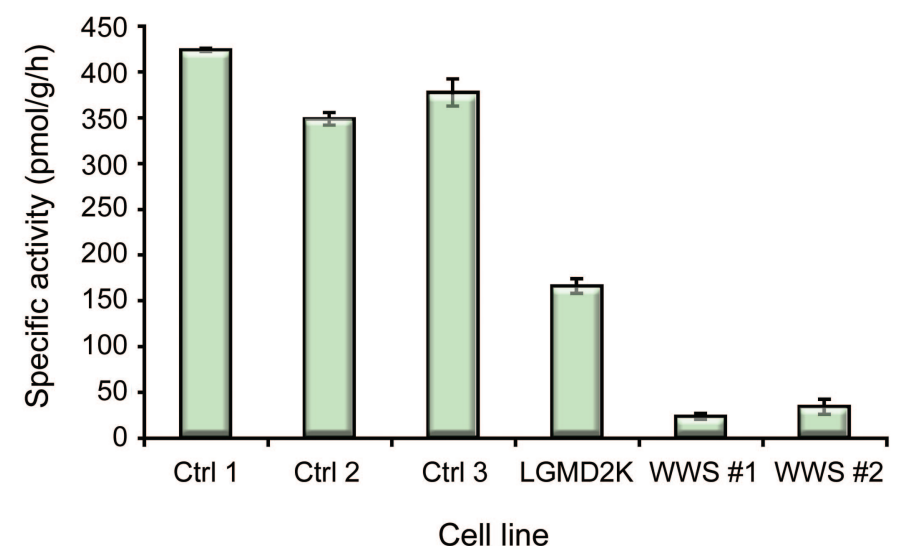

(A) Correlation of POMT enzyme activity with the amount of microsomal membranes and Dol-P-[3H]Man were recorded to determine the linear range for the POMT enzyme assay. (B) POMT activities in control cells and patient-derived dermal fibroblasts. POMT activity was based on the rate of radioactive $\left[{ }^{3} \mathrm{H}\right]$ mannose transfer from Dol-P-[$\left.{ }^{3} \mathrm{H}\right] \mathrm{Man}(125,000 \mathrm{dpm} /$ pmol) to a GST- $\alpha$-DG fusion protein.

well as in vivo O-mannosylation of $\alpha$-DG. In mammals, POMT1-POMT2 complexes are formed and complex formation is a prerequisite for full enzymatic activity. ${ }^{15,20}$ However, POMT2 might be enzymatically active in the absence of POMT1 and therefore account for the low residual in vitro activity detected ( $6 \%$ residual activity) in the WWS samples. ${ }^{21}$ Studies with protein O-mannosyltransferases (PMTs) of yeast substantiate this assumption. In yeast, Pmt $1 \mathrm{p}$ and Pmt $2 \mathrm{p}$ form heteromeric complexes; however, the individual Pmt-proteins show low levels of in vitro activity, even in the absence of their complex partner. ${ }^{22,23}$ Compared to the WWS patient, in vitro as well as in vivo O-mannosylation of $\alpha$-DG is less affected in the LGMD2K patient (figures 3 and 4).

Using dermal fibroblasts as enzyme source to measure POMT activity, we provide direct evidence that POMT activity correlates with the severity of symptoms of the analyzed dystroglycanopathy patients (figure 4). Future studies with larger patient cohorts will be necessary to corroborate our hypoth- esis of inverse correlation between residual enzymatic POMT activity in POMT1 patients and the clinical phenotype. Recently it was shown that POMT activities are abolished in lymphoblasts from various patients with POMT1 or POMT2 mutations. ${ }^{17}$ Even more, lymphoblastoid cell lines from a LGMD2K patient carrying a homozygous POMT1 pA200P mutation $^{5}$ showed almost no POMT activity as it was observed in WWS cases. ${ }^{17}$ The reported POMT activity in lymphoblastoid cells of those patients (non detectable to $\sim 0.004 \mathrm{pmol} /$ hour $/ \mathrm{mg}$ protein ${ }^{17}$ ) was at least 10 times lower compared to activities in patients' dermal fibroblasts analyzed in this study (figure 4). Thus, it is probably difficult to resolve differences in POMT activity in patient lymphoblasts. Comparative histologic analyses of muscle cross sections stained with antibodies directed toward an O-mannosidically linked glycoepitope of $\alpha$-DG showed that staining intensities correlated with the severity of the clinical phenotype of the patients. ${ }^{24,25}$ Thus, so far only indirect evidence suggested a correlation between the clinical phenotype 


\section{Figure $5 \quad$ Analysis of POMT1 protein in microsomal membrane extracts control and patient fibroblasts}

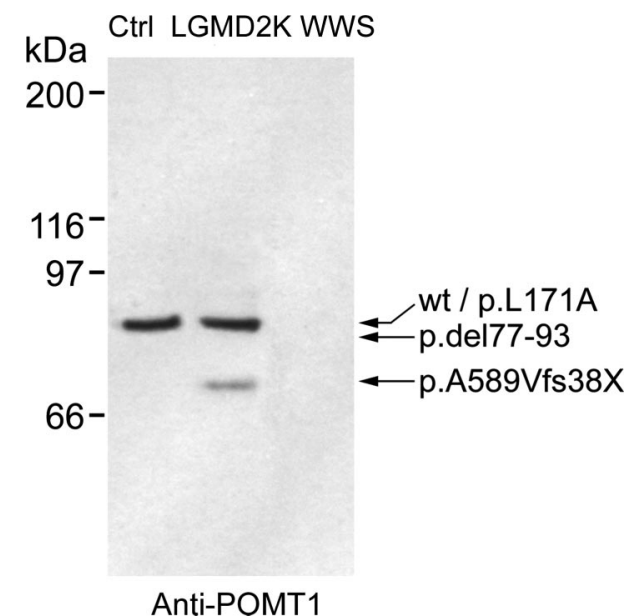

Western blots were probed with anti-POMT1 antibodies directed against the central hydrophilic domain of hPOMT1. In the LGMD2K patient, two POMT1 protein isoforms are detected as indicated. The mutant POMT1 protein in the WalkerWarburg syndrome (WWS) sample was not detectable.

and POMT activity in POMT1 and POMT2 associated $\alpha$-dystroglycanopathy patients.

Our study shows that dermal fibroblasts provide a source to detect defects caused by mutations in POMTs. This is especially important with respect to diagnosis of CMD patients. Besides POMT1 and POMT2, 4 additional genes-POMGnT1 (OMIM 606822), Fukutin-related protein (OMIM 606596), Fukutin (OMIM 607440), and LARGE (OMIM 603590) — have been established to cause secondary $\alpha$-dystroglycanopathies in humans. ${ }^{2}$ Since there is a strong overlap in the clinical features between mutations in these genes, sequencing of up to 6 genes is necessary to identify the causative mutation. Furthermore, recent studies ${ }^{9,17,26}$ demonstrated intragenic deletions and splicing abnormalities due to deep intronic mutations of POMT1 and POMT2, which have been missed by genomic sequencing of exons and flanking intronic sequences. Therefore, testing of POMT enzymatic activity provides a valuable diagnostic tool for this group of patients. Here we show that POMT activity can be measured in control and patient primary dermal fibroblasts (figure 4B). This assay represents an accurate functional method to prescreen patients harboring POMT1 and POMT2 mutations among those with suspected or confirmed $\alpha$-dystroglycanopathies. Besides the measurement of POMT activity, the use of fibroblasts will also allow the evaluation of $\alpha$-DG which is expressed in these cells (figure 3). ${ }^{27}$ Therefore, the use of dermal fibroblast cells may circumvent the need for invasive muscle biopsies when clinical findings are suggestive of secondary $\alpha$-dystroglycanopathies and exonic sequencing has not revealed pathogenic mutations.

\section{ACKNOWLEDGMENT}

The authors thank C. Endres for technical assistance. S. Strahl is a member of CellNetworks-Cluster of Excellence (EXC81).

\section{DISCLOSURE}

Dr. Lommel reports no disclosures. Dr. Cirak has received research support from the German Federal Ministry of Education and Research. Dr. Willer reports no disclosures. R. Herrmann has received research support from the German Federal Ministry of Education and Research. Dr. Uyanik reports no disclosures. Dr. van Bokhoven has received research support from Prinses Beatrix Foundation (Netherlands). Dr. Körner reports no disclosures. Dr. Voit has served on scientific advisory boards for Santhera Pharmaceuticals, Amicus Therapeutics, Inc., and AVI BioPharma; serves as Executive Editor of Neuropediatrics and an Associate Editor of Neuromuscular Disorders; may accrue revenue on patent 21512/0210136US0, filed 2009: Tricyclo-DNA antisense oligonucleotides, compositions, and methods for the treatment of disease; and has received research support from the German Federal Ministry of Education and Research. Dr. Barić has received travel expenses for lectures or educational activities not funded by industry; serves as communicating editor of the Journal of Inherited Metabolic Disease; has received honoraria from Genzyme Corporation; and receives research support from Euroglycanet and from the Ministry of Science, Education and Sports of Republic of Croatia. Dr. Hehr reports no disclosures. Dr. Strahl has received research support from the Deutsche Forschungs Gemeinschaft (grant STR443/2).

Received July 22, 2009. Accepted in final form October 29, 2009.

\section{REFERENCES}

1. Muntoni F, Brockington M, Torelli S, Brown SC. Defective glycosylation in congenital muscular dystrophies. Curr Opin Neurol 2004;17:205-209.

2. Hewitt JE. Abnormal glycosylation of dystroglycan in human genetic disease. Biochim Biophys Acta Epub 2009.

3. Lommel M, Strahl S. Protein O-mannosylation: conserved from bacteria to humans. Glycobiology 2009;19:816828.

4. Beltran-Valero de Bernabe D, Currier S, Steinbrecher A, et al. Mutations in the $\mathrm{O}$-mannosyltransferase gene POMT1 give rise to the severe neuronal migration disorder WalkerWarburg syndrome. Am J Hum Genet 2002;71:1033-1043.

5. Balci B, Uyanik G, Dincer $P$, et al. An autosomal recessive limb girdle muscular dystrophy (LGMD2) with mild mental retardation is allelic to Walker-Warburg syndrome (WWS) caused by a mutation in the POMT1 gene. Neuromuscul Disord 2005;15:271-275.

6. van Reeuwijk J, Janssen $M$, van den Elzen C, et al. POMT2 mutations cause alpha-dystroglycan hypoglycosylation and Walker-Warburg syndrome. J Med Genet 2005;42:907-912.

7. van Reeuwijk J, Maugenre S, van den Elzen C, et al. The expanding phenotype of POMT1 mutations: from Walker-Warburg syndrome to congenital muscular dystrophy, microcephaly, and mental retardation. Hum Mutat 2006;27:453-459.

8. Mercuri E, D’Amico A, Tessa A, et al. POMT2 mutation in a patient with 'MEB-like' phenotype. Neuromuscul Disord 2006;16:446-448.

9. Yanagisawa A, Bouchet C, Van den Bergh PY, et al. New POMT2 mutations causing congenital muscular dystro- 
phy: identification of a founder mutation. Neurology 2007;69:1254-1260.

10. Godfrey C, Clement E, Mein R, et al. Refining genotype phenotype correlations in muscular dystrophies with defective glycosylation of dystroglycan. Brain 2007;130: 2725-2735.

11. Biancheri R, Falace A, Tessa A, et al. POMT2 gene mutation in limb-girdle muscular dystrophy with inflammatory changes. Biochem Biophys Res Commun 2007;363:1033-1037.

12. Snijders J, Tellegen PJ, Laros JA. Snijders-Oomen Nonverbal Intelligence Test, SON-R 5 1/2-17, Manual and Research Report. Groningen: Wolters-Noordhoff; 1989.

13. Herrmann R, Straub V, Blank M, et al. Dissociation of the dystroglycan complex in caveolin-3-deficient limb girdle muscular dystrophy. Hum Mol Genet 2000;9:2335-2340.

14. Judas M, Sedmak G, Rados M, et al. POMT1-associated Walker-Warburg syndrome: a disorder of dendritic development of neocortical neurons. Neuropediatrics 2009;40:9.

15. Manya H, Chiba A, Yoshida A, et al. Demonstration of mammalian protein O-mannosyltransferase activity: coexpression of POMT1 and POMT2 required for enzymatic activity. Proc Natl Acad Sci USA 2004;101:500-505.

16. Michele DE, Barresi R, Kanagawa M, et al. Posttranslational disruption of dystroglycan-ligand interactions in congenital muscular dystrophies. Nature 2002;418: 417-422.

17. Manya $\mathrm{H}$, Bouchet $\mathrm{C}$, Yanagisawa $\mathrm{A}$, et al. Protein O-mannosyltransferase activities in lymphoblasts from patients with alpha-dystroglycanopathies. Neuromuscul Disord 2008; 18:45-51.

18. Cotarelo RP, Fano O, Raducu M, et al. A double homozygous mutation in the POMT1 gene involving exon skipping gives rise to Walker-Warburg syndrome in two Spanish Gypsy families. Clin Genet 2009;76:108-112.

19. Girrbach V, Zeller T, Priesmeier M, Strahl-Bolsinger S. Structure-function analysis of the dolichyl phosphate- mannose: protein O-mannosyltransferase ScPmt1p. J Biol Chem 2000;275:19288-19296.

20. Akasaka-Manya K, Manya H, Nakajima A, Kawakita M, Endo T. Physical and functional association of human protein O-mannosyltransferases 1 and 2. J Biol Chem 2006;281:19339-19345.

21. Willer T, Amselgruber W, Deutzmann R, Strahl S. Characterization of POMT2, a novel member of the PMT protein O-mannosyltransferase family specifically localized to the acrosome of mammalian spermatids. Glycobiology 2002;12:771-783.

22. Girrbach V, Strahl S. Members of the evolutionarily conserved PMT family of protein O-mannosyltransferases form distinct protein complexes among themselves. J Biol Chem 2003;278:12554-12562.

23. Willer T, Brandl M, Sipiczki M, Strahl S. Protein O-mannosylation is crucial for cell wall integrity, septation and viability in fission yeast. Mol Microbiol 2005;57:156170.

24. Messina S, Mora M, Pegoraro E, et al. POMT1 and POMT2 mutations in CMD patients: a multicentric Italian study. Neuromuscul Disord 2008;18:565-571.

25. Jimenez-Mallebrera C, Torelli S, Feng L, et al. A comparative study of alpha-dystroglycan glycosylation in dystroglycanopathies suggests that the hypoglycosylation of alpha-dystroglycan does not consistently correlate with clinical severity. Brain Pathol 2009;19:596-611.

26. Mercuri E, Messina S, Bruno C, et al. Congenital muscular dystrophies with defective glycosylation of dystroglycan: a population study. Neurology 2009;72:1802-1809.

27. Thompson JD, Higgins DG, Gibson TJ. CLUSTAL W: improving the sensitivity of progressive multiple sequence alignment through sequence weighting, position-specific gap penalties and weight matrix choice. Nucleic Acids Res 1994;22:4673-4680. 